Published in final edited form as:

J Chromatogr B Analyt Technol Biomed Life Sci. 2010 November 15; 878(30): 3187-3192. doi:10.1016/ j.jchromb.2010.09.017.

\title{
Determination of PF-04928473 in human plasma using liquid chromatography with tandem mass spectrometry
}

\author{
Lokesh Jain ${ }^{1,3}$, Erin R. Gardner ${ }^{2}$, Jürgen Venitz ${ }^{3}$, Giuseppe Giaccone ${ }^{4}$, Brett E. Houk ${ }^{5}$, and \\ William D. Figg ${ }^{1,3,4}$ \\ ${ }^{1}$ Clinical Pharmacology Program, Center for Cancer Research, National Cancer Institute, \\ Bethesda, Maryland, 20892 USA \\ ${ }^{4}$ Medical Oncology Branch, Center for Cancer Research, National Cancer Institute, Bethesda, \\ Maryland, 20892 USA \\ ${ }^{2}$ Clinical Pharmacology Program, SAIC-Frederick, Inc. NCI-Frederick, Frederick, Maryland, \\ 21702 USA \\ 3 Department of Pharmaceutics, School of Pharmacy, Virginia Commonwealth University, \\ Richmond, Virginia, 23298 USA \\ 5 Department of Clinical Pharmacology, Pfizer, Inc., 10578 Science Center Drive, CB10, San \\ Diego, California 92121 USA
}

\begin{abstract}
A simple, rapid and sensitive liquid chromatography/tandem mass spectrometric (LC/MS/MS) analytical method was developed for quantification of Hsp90 inhibitor PF-04928473 in human plasma, following administration of its prodrug, PF-04929113. Sample processing involved protein precipitation by addition of $0.4 \mathrm{~mL}$ of methanol containing internal standard (PF-04972487) to $50 \mu \mathrm{L}$ volume of plasma sample. Chromatographic separation of PF-04928473 and PF-04972487 was achieved on a Phenomenex ${ }^{\circledR}$ Luna C18(2) $(2.0 \times 50 \mathrm{~mm}, 5 \mu \mathrm{m})$ column using a gradient elution method with mobile phase solvents: methanol containing $0.1 \%$ formic acid and $0.1 \%$ formic acid at a flow rate of $0.25 \mathrm{~mL} / \mathrm{min}$. Detection was performed in electrospray positive ionization mode, monitoring the ion transitions from $\mathrm{m} / z$ 465.1 $\rightarrow 350.1$ (PF-04928473) and $m / z$ 447.0 $\rightarrow 329.1$ (PF-04972487). The retention times for PF-04928473 and PF-04972487 were 1.86 and 2.85 minutes, respectively. Calibration curves were generated in the range of $2-$ $2000 \mathrm{ng} / \mathrm{mL}$. The accuracy and precision ranged from $94.1-99.0 \%$ and $86.7-97.6 \%$, respectively, which were calculated using quality control samples of three different concentrations analyzed in quintuplicate on four different days.
\end{abstract}

\section{Keywords}

PF-04928473; PF-04929113; Hsp90 inhibitors; SNX-2112; SNX-5422

\footnotetext{
Author for correspondence: Dr. William D. Figg, Medical Oncology Branch, CCR, NCI/NIH, 9000 Rockville Pike, Building 10, Room 5A01, Bethesda, Maryland 20892, Tel: +1-301-402-3622, Fax: +1 301-402-8606, wdfigg@ helix.nih.gov.

Disclaimer

The content of this publication does not necessarily reflect the views or policies of the Department of Health and Human Services, nor does mention of trade names, commercial products, or organization imply endorsement by the U.S. Government.

Publisher's Disclaimer: This is a PDF file of an unedited manuscript that has been accepted for publication. As a service to our customers we are providing this early version of the manuscript. The manuscript will undergo copyediting, typesetting, and review of the resulting proof before it is published in its final citable form. Please note that during the production process errors may be discovered which could affect the content, and all legal disclaimers that apply to the journal pertain.
} 


\section{Introduction}

PF-04928473, also reported as SNX-2112, is a representative compound for a novel, small molecule class of inhibitors of the molecular chaperone heat shock protein 90 (Hsp90) (1). It is delivered via its prodrug PF-04929113 (also reported as SNX-5422), which is rapidly absorbed and converted into PF-04928473 after oral administration, Figure 1(a) $(1,2)$.

PF-04928473 selectively binds to the $\mathrm{NH}_{2}$-terminal of the ATP binding site of $\mathrm{Hsp} 90(1,2)$. Hsp90 allows proper refolding of proteins that have been destabilized, as well as conformational maturation of a set of proteins involved in intracellular signaling (3), including growth factor receptors (EGFR, PDGFR, Her2), steroid hormone receptors, kinases (Raf-1, Src etc), transcription factors, and cell cycle regulators (4). Disruption of Hsp90 results in degradation of these proteins, leading to inhibition of key signaling pathways and inhibition of tumor cell proliferation $(1,2)$. PF-04928473 has been shown to have activity against various cancers, in vitro and in vivo $(2,5,6)$. Three Phase I clinical trials of PF-04929113 are currently enrolling patients with refractory hematologic and solid tumor malignancies. To characterize the clinical pharmacokinetics of PF-04928473, a selective, reproducible, and accurate quantification method was necessary. Here, we describe the first analytical method for determination of PF-04928473 concentrations in human plasma in range of 2-2000 ng/mL. The reported method is rapid and sensitive, and is based on a simple protein precipitation approach.

\section{Methods}

\subsection{Materials and reagents}

PF-04928473 (Mol. Formula: $\mathrm{C}_{23} \mathrm{H}_{27} \mathrm{~F}_{3} \mathrm{~N}_{4} \mathrm{O}_{3}$, Purity: $99.2 \%$ by weight) and internal standard PF-04972487 (also reported as SNX-2988, Mol. Formula: $\mathrm{C}_{23} \mathrm{H}_{25} \mathrm{~F}_{3} \mathrm{~N}_{4} \mathrm{O}_{2}$, Purity: $95.8 \%$ by weight, see Figure 1(a)) were provided by Serenex, Inc. (Durham, NC, USA). Methanol (Optima grade) and formic acid (purity $\geq 98 \%$ ) were purchased from Fisher Scientific and Sigma-Aldrich, respectively. Deionized water was produced with a HydroReverse osmosis system (Durham, NC, USA) connected to a Milli-Q UV Plus purifying system (Millipore, Billerica, MA, USA). Drug-free heparinized human plasma was obtained from the Clinical Center Blood Bank at the National Institutes of Health (Bethesda, MD, USA).

\subsection{Stock solutions and standards}

The primary stock solution of PF-04928473 was prepared at concentration of $100 \mu \mathrm{g} / \mathrm{mL}$ in methanol and stored at $-20{ }^{\circ} \mathrm{C}$. Serial dilutions were performed to prepare the secondary working solutions for calibration and quality control (QC) samples. Internal standard (IS) stock solution for PF-04972487 was prepared at concentration of $100 \mu \mathrm{g} / \mathrm{mL}$ in methanol and stored at $-20^{\circ} \mathrm{C}$. The working IS solution was freshly prepared on each day of analysis by diluting the IS stock to $20 \mathrm{ng} / \mathrm{mL}$ concentration with methanol. QC samples were prepared in batch, by addition of working stocks to plasma for final concentrations of 7.5, 150 and $1500 \mathrm{ng} / \mathrm{mL}$. The $300 \mu \mathrm{L}$ aliquots of these QCs were stored at $-20{ }^{\circ} \mathrm{C}$, which were subdivided into five $50 \mu \mathrm{L}$ aliquots on the day of analysis.

\subsection{Sample preparation}

The standard calibrators were prepared by spiking $5 \mu \mathrm{L}$ of appropriate PF-04928473 working solutions to $45 \mu \mathrm{L}$ of blank human plasma in disposable glass centrifuge tubes (Kimble, Vineland, NJ). The QC samples and patient samples were thawed at room temperature, vortex mixed, and a $50 \mu \mathrm{L}$ volume was transferred to glass centrifuge tubes. To these, a $400 \mu \mathrm{L}$ volume of working internal standard solution (methanol containing IS) was 
added as a precipitation agent. These tubes were then vortex mixed for $30 \mathrm{~s}$, followed by centrifugation at $1200 \times g$ for 10 minutes. A $120 \mu \mathrm{L}$ volume of clear supernatant was then mixed with $40 \mu \mathrm{L}$ of deionized water in culture glass tubes and transferred to glass vials, of which $10 \mu \mathrm{L}$ was injected onto the column.

\subsection{HPLC-mass spectrometry apparatus and conditions}

A Waters ${ }^{\circledR}$ Acquity UPLC system was used for chromatographic separation using a Phenomenex ${ }^{\circledR}$ Luna C18 (2) column $(2.0 \times 50 \mathrm{~mm}, 5 \mu \mathrm{m})$. The autosampler and column were maintained at 4 and $35^{\circ} \mathrm{C}$, respectively. Samples were eluted using a gradient mobile phase at a flow rate of $0.25 \mathrm{~mL} / \mathrm{min}$. Mobile phases A and B were $0.1 \%$ formic acid and methanol with $0.1 \%$ formic acid, respectively. The gradient run increased linearly from $40 \%$ $\mathrm{A}$ and $60 \% \mathrm{~B}$ to $16.7 \% \mathrm{~A}$ and $83.3 \% \mathrm{~B}$ over 3.5 minutes, immediately followed by original conditions until the end of the 5.5 minute run time to re-equilibrate the column to initial conditions.

The UPLC was attached to a Waters ${ }^{\circledR}$ Micromass Quattro micro API triple quadrupole mass spectrometer equipped with an electrospray ionization source operating in positive mode. The MS analysis was carried out in multiple reaction monitoring (MRM) mode by monitoring the ion transitions from $\mathrm{m} / \mathrm{z} 465.1 \rightarrow 350.1$ for PF-04928473 and $\mathrm{m} / \mathrm{z}$ $447.0 \rightarrow 329.1$ for IS. The MS/MS conditions were as follows: capillary voltage, $3.5 \mathrm{kV}$; cone voltage, $30.0 \mathrm{~V}$; source temperature, $130{ }^{\circ} \mathrm{C}$; desolvation temperature, $410^{\circ} \mathrm{C}$; desolvation gas flow, $600 \mathrm{~L} / \mathrm{hr}$; cone gas flow, $100 \mathrm{~L} / \mathrm{hr}$, and collision energy, $23 \mathrm{eV}$. The instrumentation control and data acquisition were managed by MassLynx software (Waters ${ }^{\circledR}$ ).

\subsection{Validation procedures}

Validation was performed on 4 different days following the guidelines published elsewhere (7). On each day of analysis, two sets of calibration standards were prepared freshly at 2, 6 , $12,50,200,500,1000$, and $2000 \mathrm{ng} / \mathrm{mL}$ concentrations. One $300 \mu \mathrm{L}$ aliquot for each QC concentration $(7.5,150$ and $1500 \mathrm{ng} / \mathrm{mL}$ ) was also thawed and sub-divided into five $50 \mu \mathrm{L}$ volumes on each day of analysis. In addition, validation runs included two blank and two IS only samples.

The lower limit of detection (LOD) was the concentration for which the signal: noise ratio was at least three, across the retention window of PF-04928473. The lower limit of quantitation (LLOQ) for the assay was the lowest concentration at which PF-04928473 spiked in six different lots of plasma could be measured with accuracy greater than $80 \%$. The selectivity was assessed by analyzing the blank plasma samples from six different sources. Five replicates were run for each QC concentration, every day, for four days. The accuracy was calculated as the percent deviation of mean predicted QC concentrations from the nominal concentrations. The between- and within-run precisions were determined by one-way analysis of variance (ANOVA) using measured QC concentrations (8). The between-run precision (BRP), expressed as a percentage relative standard deviation, was defined as:

$$
B R P=\left(\frac{\sqrt{\left(\left(M S_{b e t}-M S_{\text {wit }}\right) / n\right)}}{G M}\right) \cdot 100
$$

where $\mathrm{MS}_{\text {bet }}$ and $\mathrm{MS}_{\text {wit }}$ are between- and within-run mean square variances, respectively, which were obtained from ANOVA; $\mathrm{n}$ was the number of replicate observations within each run, and GM was the grand mean. 
For each QC concentration, the within-run precision (WRP) was calculated as:

$$
W R P=\left(\frac{\sqrt{\left(M S_{w i t}\right)}}{G M}\right) \cdot 100
$$

Sample dilution was validated with QC samples of concentration $10,000 \mathrm{ng} / \mathrm{mL}$, which were diluted ten-fold with blank human plasma and processed as other QC samples. The recovery was determined as response ratio (i.e., response of analyte/IS) measured in pre-extraction spiked samples as a percentage of that measured from post-extraction spiked samples (9). The matrix effect was determined as response ratio in post-extraction spiked samples as a percentage of response ratio observed in pure solvent (9).

\subsection{Data evaluation}

Data analysis was performed with QuanLynx application, a component of MassLynx. Calibration curves were generated by least-squares linear regression analysis of the peak area ratio of PF-04928473 and IS against the nominal drug concentration using an appropriate weighting factor. The regression line was not forced through the origin $(0,0)$. The calibration curve was then used to calculate the drug concentrations in QCs and unknown samples by using the calibration equation. Concentrations falling outside the range of calibration curve can not be estimated by using this equation.

\section{Results and Discussion}

\subsection{Optimization of UPLC/MS parameters}

The MS parameters were optimized in electrospray positive ionization mode. The steps involved were: (a) Identification of parent ions for PF-04928473 and PF-04972487 in MS scan mode, determined based on maximum signal, by syringe infusion of drug solutions, (b) Tuning of MS parameters to maximize the signal for PF-04928473, the compound of interest, in MS scans. This included the optimization of cone voltage, capillary voltage, extractor voltage, desolvation temperature, desolvation gas flow, cone gas flow, resolutions, ion energies, and entrance and exit widths, (c) Identification of daughter ion for PF-04928473 by fixing the results of steps (a) and (b), i.e., $\mathrm{m} / \mathrm{z}$ value for parent ion and MS parameters, and by only adjusting the collision energy to the largest signal for daughter ion in daughter ion scan mode, (d) selection of daughter ion for internal standard PF-04972487, by keeping all the MS parameters same as for PF-04928473, except cone voltage and collision energy, which are determined separately for PF-04972487. The daughter ion scans for PF-04928473 and PF-04972487 are shown in Figure 1(b).

LC parameters were initially optimized on Waters Alliance 2695 HPLC. Isocratic and gradient methods were tested for selection of optimum LC separation conditions. Protein precipitation method was optimized for extraction of drug from plasma samples. The optimum method on Waters Alliance System was based on a gradient separation with a run time of 13 minutes. Mobile phase A and B were $0.1 \%$ formic acid and methanol with $0.1 \%$ formic acid, respectively. The gradient increased linearly from 50\% A and 50\% B to $10 \% \mathrm{~A}$ and $90 \%$ B over 6 minutes, and was held at that level up to 8 minutes. Immediately following that, it was brought to the original conditions of $50 \% \mathrm{~A}$ and $50 \% \mathrm{~B}$ until the end of the 13 minute run time, to re-equilibrate the column to initial conditions. The peak shapes after simple protein precipitation were spread out and asymmetric. To 3 parts of supernatant, 1 part of water was added, which improved the peak shapes to a narrow and symmetric one. It was thought that addition of water would increase the polarity of sample, resulting in narrow width of elution band in lipophillic C-18 column and a narrower chromatographic 
peak. Parallel to selection of mobile phases and extraction method, injection volume, needle wash and seal wash solvents were also optimized.

Switching to Acquity UPLC system, which has a significantly smaller system volume, while maintaining the gradient change rate the same, enabled us to decrease run time, including reequilibration, to 5.5 minutes, a significant decrease from the initial 13 minute run time.

\subsection{Selectivity}

No interference was observable across the retention windows for PF-04928473 and IS in blank plasma samples from six different lots of human plasma. The chromatograms for IS only and LLOQ samples are shown in Figure 2. For all blank samples, peak area was less than $5 \%$ of the area for LLOQ.

\subsection{Calibration Curve}

For the PF-04928473 concentration range of $2-2000 \mathrm{ng} / \mathrm{mL}$, the ratio of PF-04928473 to IS peak areas was fit against the nominal concentrations with a quadratic equation employing a weighting factor of $1 / \mathrm{x}^{2}$, where $\mathrm{x}$ is the nominal concentration. The mean correlation coefficient for regression equations from four days was: 0.9969 (SD: \pm 0.0005 ; range 0.9964-0.9976). The intercept for these quadratic equations was approximately close to zero with negative or positive values of 0.0006 to 0.0023 . The $\%$ deviations for calibrators from nominal concentrations were less than $2 \%$ (Supplementary Table 1). This suggests that weighted quadratic equation was adequate in describing the relationship between response ratios and nominal concentrations.

\subsection{Limits of detection and quantitation}

The LOD, based on estimated peak-to-peak noise, was determined to be $0.2 \mathrm{ng} / \mathrm{mL}$. The LOQ was estimated as $2 \mathrm{ng} / \mathrm{mL}$. For six $2 \mathrm{ng} / \mathrm{mL}$ samples prepared using plasma from different sources, percent deviation of mean measured concentration from nominal was $5 \%$ and the percent relative standard deviation (RSD) was 3\%. The mean deviation for $2 \mathrm{ng} / \mathrm{mL}$ calibrators from nominal concentration was $0.0 \%$ (RSD, 7.1\%, Supplementary Table 1). Thus, $2 \mathrm{ng} / \mathrm{mL}$ concentration exceeds the limits of accuracy and precision, and concentrations above it can be quantified with acceptable accuracy and precision.

\subsection{Accuracy and precision}

The $\%$ deviation of mean calculated QC concentrations from nominal concentrations ranged from -1 to $-6 \%$ (Table 1) at three concentrations of 7.5, 150 and $1500 \mathrm{ng} / \mathrm{mL}$, which were well within the acceptable limits of $\pm 15 \%$, suggesting that assay method accurately estimated PF-04928473 plasma concentrations. The between-run imprecision ranged from 11.2 to $13.3 \%$ and within-run imprecision was between $2.4-3 \%$, which were also within the acceptable limits of $\pm 15 \%$ and indicates that assay predictions were precise. The inaccuracy and between- and within-run imprecisions for dilution analysis were $3.9 \%, 11.2 \%$ and $2.7 \%$, respectively, all below the acceptable limits of $\pm 15 \%$. This confirms that samples with concentrations above the upper limit of quantification can be analyzed using ten-fold dilution.

\subsection{Recovery and matrix effect}

Recovery and matrix effects were checked at three concentrations, 6, 200 and $2000 \mathrm{ng} / \mathrm{mL}$. Recovery was determined by comparison of response ratio for the samples with preextraction and post-extraction addition of PF-04928473. Recovery ranged from 99\% to $113 \%$ for studied concentrations (Supplementary Table 2). Matrix effect was calculated by comparison of response ratio for sample with post-extraction addition of PF-04928473 to 
that of response for PF-04928473 spiked in water. Matrix effect was less than $11 \%$ for the studied concentrations (Supplementary Table 2).

\subsection{Freeze-thaw stability}

Three sets of QC samples at were subjected to one, two and three freeze-and-thaw cycles, respectively. Each freeze cycle lasted for more than $15 \mathrm{hrs}$. All these samples were analyzed on the same day along with freshly prepared QC samples. The \% change in measured concentrations after each freeze-thaw cycles was less than $10 \%$ (Table 2), indicating that repeated exposure to freeze-thaw cycles does not degrade PF-04928473 in human plasma through at least three cycles.

\subsection{Autosampler, short-term and long-term stability}

An entire set of samples was left in autosampler at $4{ }^{\circ} \mathrm{C}$ for $24 \mathrm{hrs}$ after initial run, and was subsequently reanalyzed. Comparison of measured concentrations after $24 \mathrm{hr}$ with initial concentrations showed less than 3\% deviation (Table 2). This suggests that when necessary, samples can be reanalyzed the following day without compromising on the measurements, if stored properly.

The short-term or "bench-top" stability of PF-04928473 working stock solutions were assessed at three concentrations: 6, 200 and $2000 \mathrm{ng} / \mathrm{mL}$. Three samples at each concentration were left on bench-top at room temperature and a set of similar samples was stored at $-20^{\circ} \mathrm{C}$. After $10 \mathrm{hrs}$ these samples were analyzed in the same run. The $\%$ change at studied concentrations was less than $2 \%$, suggesting that integrity of PF-04928473 in methanol solvent was not affected for at least $10 \mathrm{hrs}$ when stored at room temperature. For long-term stability, the QC samples stored at $-20^{\circ} \mathrm{C}$ for 51 days were compared with freshly prepared QCs. The percentage deviation at 7.5, 150 and $1500 \mathrm{ng} / \mathrm{mL}$ concentrations was less than $6 \%$, suggesting that samples integrity is not compromised when stored at -20 ${ }^{\circ} \mathrm{C}$ for at least 51 days.

\section{Application}

The validated method was used to determine the plasma concentrations of PF-04928473 in a phase I dose escalation study, enrolling patients with refractory solid tumor malignancies and lymphomas. In this trial, blood samples were collected up to $48 \mathrm{hrs}$, in $7 \mathrm{~mL}$ sodium heparin tubes, and plasma was separated by centrifugation for 5 minutes at $1200 \times \mathrm{g}$ and 4 ${ }^{\circ} \mathrm{C}$. The plasma samples were stored at $-80^{\circ} \mathrm{C}$ prior to analysis. A typical plasma concentration - time profile for a patient receiving $33 \mathrm{mg} / \mathrm{m}^{2} \mathrm{PF}-04929113$ is shown in Figure 3.

\section{Conclusion}

In conclusion, a novel bioanalysis method was developed and validated for determination of PF-04928473 in human plasma using tandem mass spectrometric detection. The described method is simple, rapid, selective and sensitive and can be easily implemented into routine practice. The method gives accurate and precise measurements of PF-04928473 plasma concentrations with a small plasma volume of $50 \mu \mathrm{L}$ and in a short run time of 5.5 minutes. The results for validation parameters such as recovery, matrix effect and stability following freeze-thaw cycles, at room temperature and after long-term were within the acceptable limits. This method is currently being used for analysis of plasma samples from a dose escalation trial in patients with solid tumors and lymphomas. 


\section{Supplementary Material}

Refer to Web version on PubMed Central for supplementary material.

\section{Acknowledgments}

\section{Financial Support}

This project has been funded in whole or in part with federal funds from the National Cancer Institute, National Institutes of Health, under contract HHSN261200800001E (ERG). This work was supported by the Intramural Research Program of the NIH, National Cancer Institute, Center for Cancer Research.

\section{References}

1. Okawa Y, Hideshima T, Steed P, et al. SNX-2112, a selective Hsp90 inhibitor, potently inhibits tumor cell growth, angiogenesis, and osteoclastogenesis in multiple myeloma and other hematologic tumors by abrogating signaling via Akt and ERK. Blood. 2009; 113:846-55. [PubMed: 18948577]

2. Chandarlapaty S, Sawai A, Ye Q, et al. SNX2112, a synthetic heat shock protein 90 inhibitor, has potent antitumor activity against HER kinase-dependent cancers. Clin Cancer Res. 2008; 14:240-8. [PubMed: 18172276]

3. Neckers L, Ivy SP. Heat shock protein 90. Curr Opin Oncol. 2003; 15:419-24. [PubMed: 14624223]

4. Maloney A, Workman P. HSP90 as a new therapeutic target for cancer therapy: the story unfolds. Expert Opin Biol Ther. 2002; 2:3-24. [PubMed: 11772336]

5. Hall, SE.; Barabasz, A.; Barta, T., et al. Chemoproteomics-driven drug discovery: identification of novel Hsp90 inhibitors. AACR Annual Meeting; Los Angeles, CA. April 14-17, 2007; 2007.

6. Steed P, Huang H, Fadden P, et al. SNX-2112: a novel, selective, potent small molecule inhibitor of Hsp90 with unique pharmacodynamic properties. Eur J Cancer. 2006; 4:165.

7. The Unites States Department of Health and Human Services, Guidance for Industry: Bioanalytical Method Validation. Food and Drug Administration, Center of Drug Evaluation and Research and Center for Veterinary Medicine. May 2001 BP.

8. Polettini, A., editor. Applications of LC-MS in Toxicology. Pharmaceutical Press; London, UK: 2006. p. 81

9. Jain L, Gardner ER, Venitz J, Dahut W, Figg WD. Development of a rapid and sensitive LC-MS/ MS assay for the determination of sorafenib in human plasma. J Pharm Biomed Anal. 2008; 46:362-7. [PubMed: 18309574] 
<smiles>[R]OC1CCC(Nc2cc(-n3nc(C)c4c3CC(C)(C)CC4=O)ccc2C(N)=O)CC1</smiles><smiles>CC1(C)CC(=O)c2c(CF)nn(-c3ccc(C#N)c(N[C@H]4CC[C@H](O)CC4)c3)c2C1</smiles>
PF-04929113 R=C(O)CH $\mathrm{CH}_{2}$

Figure 1 (a)

\section{PF-04972487}




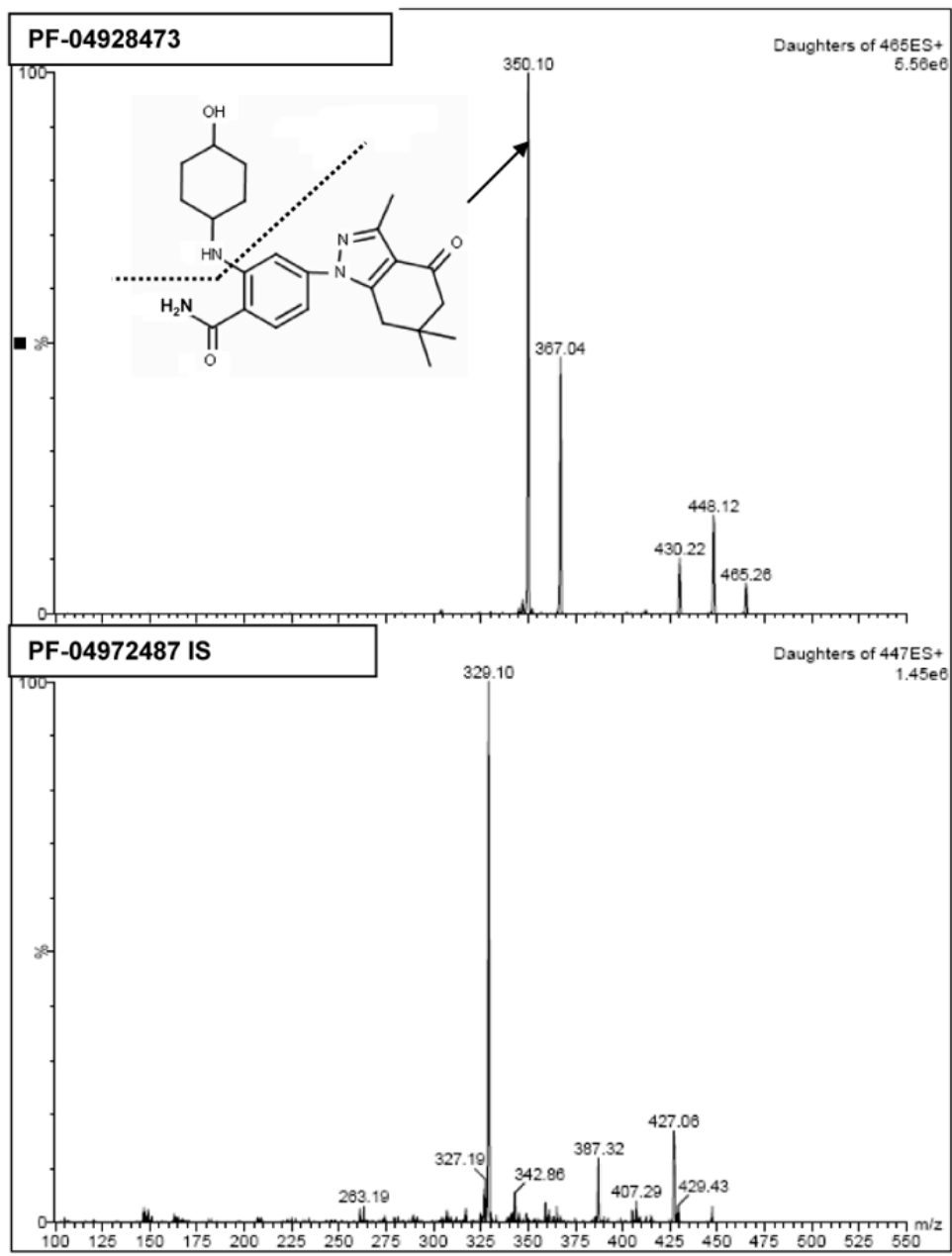

Figure 1 (b)

Figure 1.

Figure 1(a) Chemical structures of PF-04929113 (pro-drug), PF-04928473 (active molecule), and PF-04972487 (internal standard).

Figure 1(b). Full scan product ion spectra of PF-04928473 and PF-04972487 


\section{A PF-04928473 \\ IS only}

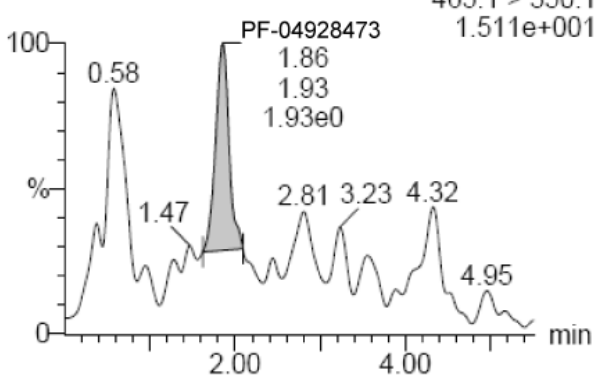

B PF-04928473

PF-04928473 $2 \mathrm{ng} / \mathrm{mL}$ MRM of 2 channels, ES+

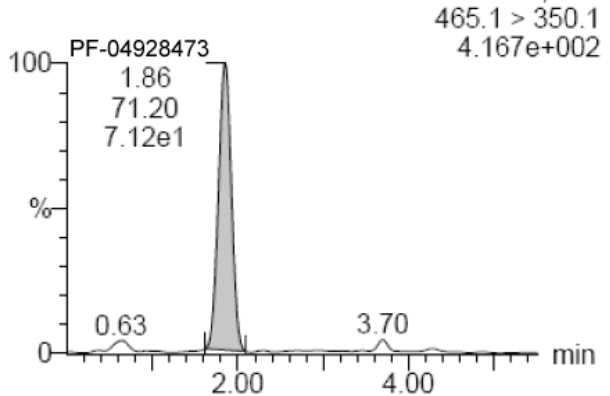

PF-04972487

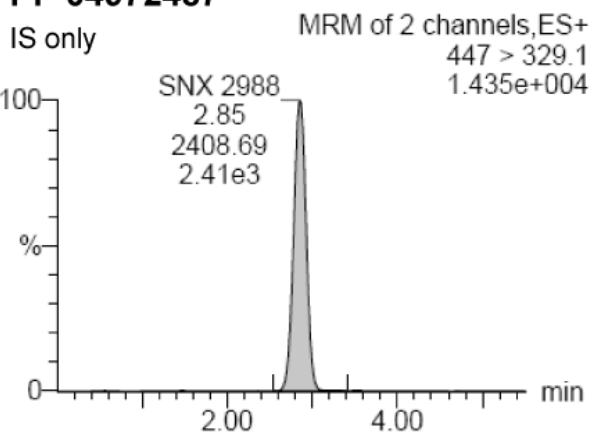

\section{PF-04972487}

PF-04928473 $2 \mathrm{ng} / \mathrm{mL}$ MRM of 2 channels, ES+

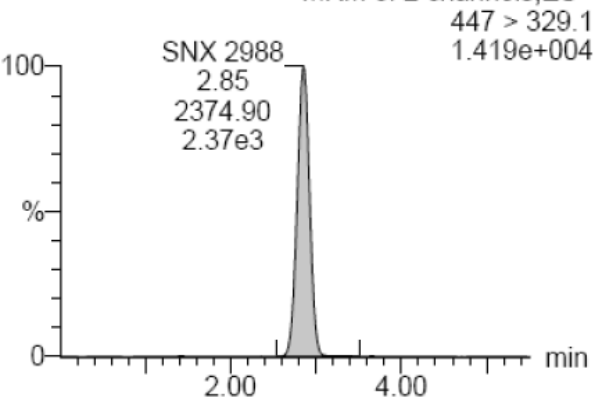

Figure 2.

Typical chromatograms showing the $\mathrm{m} / \mathrm{z} 465.1 \rightarrow 350.1$ (PF- 04928473) and $\mathrm{m} / \mathrm{z}$ $447.0 \rightarrow 329.1$ (PF-04972487, IS) transitions for (A) a blank human plasma sample with IS, and (B) an LLOQ (2 $\mathrm{ng} / \mathrm{mL})$ calibrator sample with IS. 


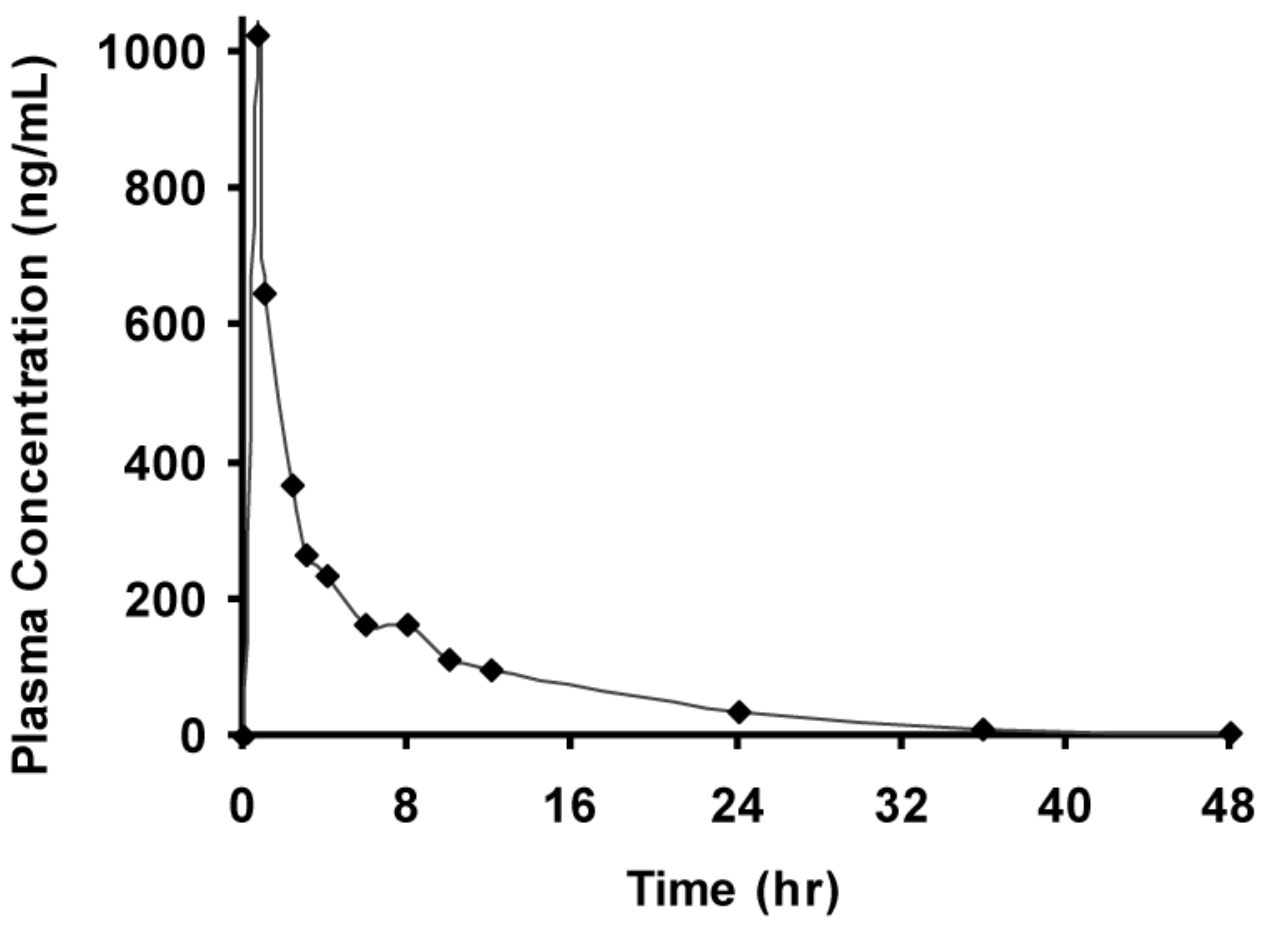

Figure 3.

Typical plasma concentration - time profile for PF- 04928473 from a patient with cancer following treated with a single oral dose of $33 \mathrm{mg} / \mathrm{m}^{2} \mathrm{PF}-04929113$. 
Table 1

Intra-run and inter-run accuracy and precision from quality control samples

\begin{tabular}{|c|c|c|c|c|}
\hline & \multicolumn{4}{|c|}{ PF- 04928473 nominal concentration $(\mathrm{ng} / \mathrm{mL})$} \\
\hline & $7.5(\mathrm{~N}=20)$ & $150(\mathrm{~N}=20)$ & $1500(N=20)$ & $10,000$ (dilution $10 \times)(\mathrm{N}=15)$ \\
\hline Mean & 7.4 & 141.1 & 1476.4 & 10380 \\
\hline $\operatorname{RSD}(\%)$ & 5.6 & 5.8 & 5.0 & 5.8 \\
\hline $\operatorname{DEV}(\%)$ & -1.0 & -5.9 & -1.6 & 3.9 \\
\hline \multicolumn{5}{|l|}{ Imprecision } \\
\hline Within-run & 2.7 & 2.9 & 2.4 & 2.7 \\
\hline Between-run & 12.4 & 13.3 & 11.2 & 11.2 \\
\hline
\end{tabular}

RSD: relative standard deviation, DEV: percent deviation from nominal value 
